\title{
The Salmonella effector AvrA mediates bacterial intracellular survival during infection in vivo
}

\author{
Huixia Wu, Rheinallt M. Jones, and Andrew S. Neish ${ }^{*}$ \\ Epithelial Pathobiology Unit, Department of Pathology and Laboratory Medicine, Emory University \\ School of Medicine, Atlanta GA, 30322
}

\section{SUMMARY}

The enteric pathogen Salmonella typhimurium secretes the preformed AvrA effector protein into host cells. This acetyltransferase has been shown to modulate mammalian intestinal immune and survival responses by inhibition of JNK MAPK. To study the role of this effector in natural enteric infection, we used a mouse model to compare wild type Salmonella typhimurium to an isogenic AvrA null Salmonella mutant. Salmonella lacking AvrA induced increased intestinal inflammation, more intense systemic cytokine responses, and increased apoptosis in epithelial cells. Increased apoptosis was also observed in extra epithelial macrophages. AvrA null infected mice consistently showed higher bacterial burden within mucosal lymphoid tissues, spleen and liver by 5 days post infection, which indicated a more severe clinical course. To study the molecular mechanisms involved, recombinant adenoviruses expressing AvrA or mutant AvrA proteins were constructed, which showed appropriate expression and mediated the expected inhibition of JNK signaling. Cultured epithelial cells and macrophages transduced with AvrA expressing adenovirus were protected from apoptosis induced by exogenous stimuli. In conclusion, the results demonstrated that Salmonella AvrA modulates survival of infected macrophages likely via JNK suppression, and prevents macrophage death and rapid bacterial dissemination. AvrA suppression of apoptosis in infected macrophages may allow for establishment of a stable intracellular niche typical of intracellular pathogens.

\section{Keywords}

Salmonella; AvrA; JNK; Apoptosis; Innate immunity

\section{INTRODUCTION}

Salmonella typhimurium is a food-borne Gram-negative pathogen that is a major public health challenge both in the US and the developing world. Infection is generally confined to the intestinal mucosa and is usually self-limited, though systemic dissemination may occur in immunocompromised persons. Characteristically, intestinal Salmonellosis shows marked acute neutrophilic inflammation without development of acute epithelial destruction typically associated with other enteric pathogens such as Shigella infection (Sansonetti and Di Santo, 2007), or macrophage cell death that occurs in Yersinia infection (Ruckdeschel et al., 1997). The pathogenesis of Salmonella infection has been extensively studied in mammalian model systems. After ingestion and gastric passage, Salmonella reaches the small intestine lumen where it translocates epithelia overlaying mucosal lymphoid aggregates, or Peyer's patches (PP) or invades across the absorptive epithelium of the villus

"Corresponding author: Andrew S. Neish, M.D., Department of Pathology, Emory University School of Medicine, Room 105F, Whitehead Bldg., 615 Michael Street, Atlanta GA, 30322, Tel: (404) 727-8545, Fax: (404) 727-8538, aneish@emory.edu. 
units (Monack et al., 2000). Alternatively, Salmonella may be taken up directly by lamina propria (LP) dendritic cells (DCs) that interdigitate between epithelial cells and sample the luminal content (Tam et al., 2008). In either case, the bacteria establish itself within dendritic cells or macrophages in the PP or LP, and Salmonella then rapidly disseminate to phagocytic cells of the hepatic and splenic reticuloendothelial system, ultimately taking up residence in macrophages and macrophage-like cells. These events stimulate inflammatory signaling, including activation of the NF-KB and MAPK pathways, resulting in secretion of inflammatory mediators, accumulation of neutrophils and development of systemic inflammatory syndrome (Tam et al., 2008).

In order to invade and survive with host cells, Salmonella possesses a range of effector proteins that are translocated into the host cells via a type III secretion apparatus (Staskawicz et al., 2001). These effector proteins are generally assumed to influence host cellular functions to facilitate Salmonella invasion and intracellular carriage. Given the central role innate immune pathways play in the control of enteric pathogens, it is not surprising that diverse bacterial pathogens have evolved mechanisms to surmount their effects. Inhibition of MAPKs (and NF- $\mathrm{kB}$ ) has been described in Yersinia, Shigella and enteropathogenic E. coli infections (Li et al., 2007; Orth et al., 1999; Duesbery et al., 1998). It is generally accepted that bacterial pathogens evolved these capacities to prevent or reduce the upregulation of the inflammatory response, thus blocking the recruitment of phagocytic leukocytes. Acute infections may also involve apoptotic killing of epithelial cells and macrophages -events likely permissive for bacterial proliferation and dissemination- while chronic intracellular pathogens may suppress apoptosis as a mechanism to maintain a stable niche.

A relevant example of a secreted factor with immunosuppressive function is AvrA, a $33 \mathrm{kDa}$ Salmonella protein that is translocated into intestinal epithelial cells during the initial stages of invasion (Hardt and Galan, 1997). AvrA is a member of a family of acetyltransfereases expressed in several enteric pathogens, which includes YopJ/P in Yersinia pseudotuberculosis (Orth et al., 1999) and VopP in Vibro parahemalyticus (Mukherjee et al., 2007). We have shown that when AvrA is overexpressed in transfected cells and in a Drosophila transgenic model, activation of the MAPK JNK and consequent transcriptional activation of a range of inflammatory effector genes is blocked (Jones et al., 2008; CollierHyams et al., 2002). Du and Galan demonstrated remarkably similar suppressive effects of AvrA on JNK signaling in a yeast model (Du and Galan, 2009). Interestingly, in both flies and human cells, innate immune signaling blockade occurred without induction of apoptotic cell death characteristically seen during inhibition of host inflammatory signaling pathways. Consistently, infection of mice with mutant Salmonella lacking AvrA $\left(a v r A^{-}\right)$resulted in increased injury in the gut (Jones et al., 2008). In this report, we extended these in vivo findings in a well established murine model of enteric and systemic Salmonellosis. We show that Salmonella SL3201 $\left(\mathrm{avrA}^{-}\right)$induced higher levels of inflammatory mediator expression, leukocyte recruitment, cellular inflammation and apoptosis in epithelia and monocytic cells of the mucosa and systemic lymphoid tissue. We demonstrate that Salmonella SL3201 $\left(a v r A^{-}\right)$was able to proliferate more efficiently in mesenteric lymph node (MLN) and spleen. Despite the increased proliferation by mutant Salmonella, we demonstrate these avrA $A^{-}$organisms do not maintain the typical intracellular niche characteristic of w.t. Salmonella. Consistently, we show that AvrA is a potent inhibitor of macrophage apoptosis, and can suppress cell death induced by extraneous signals. These data suggest that the AvrA effector can control mucosal and systemic innate immune and apoptotic responses during natural infection, thus elucidating a mechanism of how an intracellular pathogen can deflect both inflammatory and apoptotic defenses. 


\section{RESULTS}

\section{Natural infection with mutant Salmonella SL3201 (avrA $\left.{ }^{-}\right)$induces a more severe inflammatory response}

To study the role of AvrA in natural intestinal infection in vivo, we employed the well documented streptomycin pretreatment mouse model of enteric Salmonellosis (Vijay-Kumar et al., 2006; Barthel et al., 2003). Mice were infected with Salmonella typhimurium parent strain SL3201, hereafter referred to as wild type w.t. SL3201, or two previously characterized isogenic SL3201 mutants, including a non-polar insertional mutation in the chromosomal avrA gene SL3201( $\left(\mathrm{vrr}^{-}\right)$, and the SL3201( $\left.a v r A^{-}\right)$strain complemented with a plasmid harboring wild type AvrA SL3201(avrA-/pAvrA) (Collier-Hyams et al., 2002). Four groups of female Balb/cj mice were pretreated with streptomycin for 24 hours before infection with $10^{8} \mathrm{CFU} /$ mouse Salmonella by oral gavage, and sacrificed at intervals post infection (p.i.). To compare the inflammatory response, we evaluated cecal histopathology, which was graded with a semi quantitative scale (Vijay-Kumar et al., 2006). The earliest morphological changes were seen at 6 hours p.i., manifested by loss of goblet cells, polymorphonuclear cell infiltration into the lamina propria, and epithelial damage (Fig. 1A). Histopathological changes peaked at 24 hours p.i. Most notably, infection with SL3201 $\left(a v r A^{-}\right)$resulted in a marked increase in the proinflammatory response at 24 hours p.i, relative to the parent or complemented strain (Fig. 1A and 1B). Grossly, infection with SL3201 $\left(\mathrm{avrA}^{-}\right)$resulted in inflammatory exudate and massive edema in three out of five mice at 6 hours p.i. These effects were not seen following infection with w.t. SL3201 strain or complemented SL3201 ( $a v r A^{-} / \mathrm{pAvrA}$ ) (data not shown). Consistently, cecal myeloperoxidase (MPO) activity was significantly elevated following infection with SL3201 $\left(\mathrm{avrA}^{-}\right)$at $6,12,24$, and $48 \mathrm{~h}$ p.i., compared to the control strains (Fig. 1C). These data corresponded closely with disease index scores presented in Figure 1B. Importantly, in each assay, the increased inflammation induced by SL3201 $\left(\mathrm{avrA}^{-}\right)$was not detected following infection by the complemented SL3201( $\left.a v r{ }^{-} / \mathrm{pAvrA}\right)$ strain, together showing that AvrA functions in vivo to suppress intestinal inflammation up to 48 hours following Salmonella typhimurium infection (Fig. S1).

\section{Salmonella secreted protein AvrA dampens apoptosis in multiple murine cell lines following infection}

We next investigated the effects of AvrA on apoptosis in mucosal cell types following Salmonella infection. We previously reported that infection of mice with SL3201 (avrA $\left.{ }^{-}\right)$ induced significantly increased numbers of apoptotic epithelial cells at 6 hours p.i. compared to w.t. SL3201 or SL3201 (avrA-/pAvrA) (Jones et al., 2008). Here, we expand on these observations and show that SL3201 ( $a v r A^{\top}$ ) also induces elevated levels of apoptotic cells in the cecal mucosa at 24 hours following infection (Fig. S2A and S2B). We assessed the level of apoptosis in mucosal tissue or in the spleen at the indicated time points because they are consistent with the progression of Salmonella invasion and dissemination following infection. Importantly, in the course of these experiments we also observed a similar pattern of increased SL3201 ( $\mathrm{avrA}^{-}$)-induced apoptosis in cecal lymphoid tissues located in subepithelial compartments (Fig. 2A and 2B). Immunostaining of these tissues revealed that the apoptotic cells also stained positive with the macrophage marker F4/80 (Fig. S2C). To confirm these findings in vitro, we infected murine bone marrow derived macrophages or non-transformed primary epithelial cell monolayers (rat IEC-6 cells) with the Salmonella strains. Consistent with the in vivo observations, SL3201 (avrA ${ }^{-}$) induced apoptosis in significantly increased numbers of bone marrow derived macrophages (Fig. 2C) or primary epithelial cells (Fig. 2D) compared to the w.t. or complemented strains. Thus, in vitro and in vivo, SL3201(avrAך) induced markedly increased cell death, suggesting a physiological role 
for AvrA in suppressing cell death pathways in both cell types involved in Salmonella infection.

\section{Salmonella SL3201 (avrA-)exhibited increased mucosal and systemic dissemination in vivo}

Induced apoptosis in infected epithelial cells and macrophages is seen in certain Shigella and Yesinia infections and is associated with rapid dissemination, while suppression of apoptosis is commonly seen in long term intracellular parasites (Weinrauch and Zychlinsky, 1999). To determine the effect of apoptosis in model Salmonella infection we investigated local and systemic dissemination up to 48 hours p.i. Using the infection model described in Figure 1, bacterial colonization was enumerated in different organs and tissues at regular intervals. Significantly higher numbers of Salmonella SL3201( $\mathrm{avrA}^{-}$) colonized cecal wall tissue, including Peyer's patches (PP) and mesenteric lymph nodes (MLN) compared to complemented Salmonella strains (Fig. 3A-C). No Salmonella were found in PBS treated mice. At 24 hours p.i., Salmonella were disseminated to the spleen of infected groups, again with SL3201 (avrA ${ }^{-}$) exhibiting higher levels of colonization (Fig. 3D). By seven days, increased weight loss became apparent in the mice infected with SL3201 (avrA ${ }^{`}$ (Fig. 3E), and by 12 days increased mortality was apparent (Fig. 3F). All mice were sacrificed at that time point because of clinical decline. Necropsy at the seven day time point was remarkable for gross hepatosplenomegy (Fig. 3G) and increased systemic cytokine concentrations (Fig. $3 \mathrm{H})$. Together, these data show that loss if Salmonella AvrA function results in increased infection of gut lymphoid tissues, and increased systemic dissemination during pathogenesis.

\section{SL3201 $\left(\mathrm{avrA}^{-}\right)$does not establish an intracellular niche within macrophages}

Data presented in Figure 3 indicated AvrA dependent differences in the bacterial burden in systemic lymphoid tissues. To investigate this further, we used a three or four color flow cytometric method to study intracellular Salmonella in macrophages. Whole spleens from mice infected with w.t. SL3201, SL3201(avrA 7 ) or with SL3201 ( $\left.a v r A^{-} / \mathrm{pAvrA}\right)$ for 6 days were dissociated, stained with the macrophage surface marker (F4/80), fixed, permeabilized, and stained for intracellular Salmonella using an anti-Salmonella antibody (KPL Inc., Gaithersburg, MD). Using this technique, we observed significantly reduced numbers of macrophages-like cells harboring intracellular SL3201 ( $\left.a v r A^{-}\right)$, compared to w.t SL3201 or SL3201 ( $a v r A^{-} /$pAvrA) (Fig. 4A and B). We detected considerably less numbers of SL3201 ( $a v \mathrm{rA}^{-} / \mathrm{pAvrA}$ ) within splenic macrophages compared to the wild type SL3201. We suspect that extended infection in the absence of antibiotic pressure might have resulted in the eviction of the complement pAvrA plasmid. Nevertheless, the number of SL3201 (avrA ${ }^{-}$/ pAvrA) detected within the spleen was still significantly more than the numbers of SL3201 $\left(\right.$ avr $\left.^{\top}{ }^{\top}\right)$ detected. These observations correlated with increased total bacterial load detected within the spleen at 6 days p.i., with SL3201 $\left(a v r A^{-}\right)$infection resulting in several order of magnitude higher spleen bacterial load compared to w.t. or complemented controls (Fig. 4C). To confirm that w.t SL3201 or SL3201 ( $a v r{ }^{-} / \mathrm{pAvrA}$ ) establish an intracellular niche, we undertook in vivo gentamicin protection assay (Glomski et al., 2003). Gentamicin only targets extracellular bacteria, thus intracellular bacteria will be protected against its microbicidal activity. Consistent with observations from flow cytometry analysis described in figure 4A and B, w.t SL3201 or complemented SL3201(avrA ${ }^{-}$pAvrA) strains were protected from gentamicin treatment, whereas a significant decrease in the population of SL3201( $\left(a v A^{-}\right.$) within the spleen was detected (Fig. 4D). We also detected a significant decrease in the number of SL3201 $\left(\mathrm{avrA}^{-}\right)$present in mucosal tissues including the Peyers Patch, MLN and Ceacum following gentamycin treatment (Fig. S3). Together, these data show that a significant number of SL3201( $a v r A\urcorner$ ) do not occupy an intracellular niche following infection. 


\section{AvrA directly suppresses induced apoptosis}

Based on our observation in cecal lymphoid aggregates (Fig. 2A), we hypothesized that the decreased number of SL3201 ( $\left.a v r A^{-}\right)$occupying an intracellular niche was as a resulted of increased macrophage apoptosis. To investigate this possibility, we performed immunohistochemical staining on spleens of infected mice at 7 days p.i. and quantified apoptotic cells. We detected significantly elevated numbers of active caspase 3 positive macrophage-like cells in the spleens of mice infected with SL3201( $\left.\mathrm{avrA}^{-}\right)$, but not within spleens of mice infected with w.t SL3201 (Fig. 5A). These data prompted us to assess the extent to which AvrA protein in isolation could directly protect cells from apoptosis. We constructed adenoviral vectors expressing vector alone, wild type AvrA, or a mutant and catalytically inactive version of AvrA (mAvrA). The adenoviral vectors were packaged, and transduced into primary polarized epithelial cells, or into bone marrow derived macrophages (BMDM); both cell lines which are otherwise not easily transfected by plasmid DNA (Fig. S4). AvrA has been shown to be a potent inhibitor of JNK MAPK in yeast, Drosophila and mammalian cell systems (Du and Galan, 2009; Jones et al., 2008; Collier-Hyams et al., 2002). We first confirmed that this activity was present in AvrA expressed from adenoviral particles. AD293 cells were transduced with the adenoviral particles harboring vector control, AvrA, or mAvrA, and stimulated with rhTNFa for 15 minutes. Immunoblot analysis showed that Ad-AvrA completely inhibited TNFa induced JNK phosphorylation, but vector alone and mutant AvrA did not (Fig. S5). We then transduced AvrA expressing adenoviruses into primary epithelial IEC- 6 cells and treated cells with $0.5 \mu \mathrm{g} / \mathrm{ml}$ of Actinomycin D and $100 \mathrm{ng} / \mathrm{ml}$ of TNF $\alpha$ for $24 \mathrm{~h}$ to induce apoptosis. We had previously used this cell line and treatment to evaluate the anti-apoptotic effect of TLR ligands (VijayKumar et al., 2006; Jones et al., 2011). The culture supernatants of the transduced cells were collected to assay the level of the lactate dehydrogenase (LDH), a soluble cytosolic enzyme released into the culture medium following loss of membrane integrity resulting from either apoptosis or necrosis. The LDH assays showed that Actinomycin D together with TNF $\alpha$ induced apoptosis in IEC- 6 cells, and that AvrA but not mAvrA inhibited induced apoptosis in cultured epithelial cells (Fig. 5B). Additionally, immunoblot analysis of equivalent amounts of cellular protein lysates using antibodies against cleaved PARP confirmed that AvrA suppressed apoptosis in primary epithelial cells (Fig. 5C), and in murine bone marrow derived macrophages (Fig. 5D). Taken together, these data indicated that the AvrA protein, in isolation, can not only suppress the stress signaling JNK pathway, but can actively block cellular apoptosis stimulated by exogenous agonists.

\section{DISCUSSION}

In this report, we demonstrate that Salmonella mutants lacking AvrA provoke an aggravated disease. Salmonella is an intracellular pathogen that transiently invades epithelial cells and then takes up residence within a vacuole in monocytic cells (Monack et al., 2004). While infected cells can progress to apoptosis and do activate typical proinflammatory responses, our data reveal the AvrA protein dampens both of these host reactions - presumably to establish a stable intracellular niche (and likely avoid adaptive immunity). Loss of AvrA caused increased apoptosis in both epithelial and macrophage cellular hosts during Salmonella infection and was simultaneously associated with loss of intracellular carriage and increased microbial parenchymal burden in systemic lymphoid tissues in the later stages of systemic infection, eventuating in increased mortality.

In past reports, we showed that AvrA acts as an acetyltransferase modifying critical serine and threonine residues of target MKK4 and 7 and thereby blocking both phosphorylation and activation of these proteins and downstream JNK signaling (Jones et al., 2008). This specificity of AvrA action on MKK4 has also been shown in a yeast system (Du and Galan, 2009). Homologs of AvrA have been described in other enteric pathogens including Yersinia 
pseudotuberulosis protein YopJ/P (Orth et al., 1999), Vibrio parahemalyticus protein VopA (Trosky et al., 2007; Trosky et al., 2004), and Aeromonas salmonicida protein AopP (Fehr et al., 2006), as well as in plant pathogens (Ellis et al., 2007). These effectors have been described to possess inhibitory acetyltransferase activities toward various MAPKK family and IKKs, and because of the diversity of substrates they have different effects on infected cells, but are generally proapoptotic (Jones and Neish, 2011; Trosky et al., 2007; Mittal et al., 2006; Mukherjee et al., 2006). Specifically, YopJ has a broad range of inhibitory activities against ERK, p38, JNK, and IKK pathways (Orth et al., 1999). VopA has been shown to inhibit ERK, p38 and JNK, but not IKK (Trosky et al., 2004), and AopP is apparently specific to the NF- $\mathrm{KB}$ pathway (Fehr et al., 2006). YopJ and AopP are both associated with rapid apoptosis of infected cells (Jones et al., 2008; Orth et al., 1999). Furthermore, in plants, members of this protein family were originally designated "Avr" proteins that mediate "avirulence" functions in plants, pathologically manifested by local apoptosis of the infected plant cells. However, the absence of the avirulence gene resulted in systemic bacterial dissemination, and plant death (Staskawicz et al., 2001). Such a role was originally hypothesized for AvrA (hence the nominal designation) (Hardt and Galan, 1997). However, we continue our striking finding that AvrA, unique among this family of acetyltransferase effectors, can potently suppress apoptosis. AvrA virally transduced into cells showed blockade of exogenous inducers of apoptosis. These data also indicate that AvrA can meditate its biochemical functions in isolation, without translocated bacterial cofactors or chaperones, although recent studies show that AvrA may require phosphorylation before it is enzymatically active (Du and Galan, 2009). In the context of proinflammatory enteropathogenic Salmonellosis, our results suggest AvrA represses apoptosis during multiple stages of the infectious process, presumably as a mechanisms of maintaining its intracellular niche. Salmonella induces a brisk but self-limited acute inflammatory response in the epithelia, both in natural human and model murine infections, without the overt epithelial destruction more characteristic of dysenteric clinical syndromes caused by Shigella or enterohemorrhagic E. coli infection (Sansonetti and Di Santo, 2007). Our murine experiments with Salmonella avrA ${ }^{-}$mutant resulted in increased cellular inflammation and a massive increase in epithelial apoptosis, reminiscent of Shigellosis. The increased macrophage apoptosis caused by the Salmonella ( $\mathrm{avrA}^{-}$) mutant allowed increased dissemination into extracellular tissue, associated with increased systemic disease. Furthermore, increased epithelial cell apoptosis induced by infection with SL3201( $a v r A^{\top}$ ) resulted in increased total numbers of invading and disseminating bacteria, as reflected in the increase numbers of SL3201 $\left(\mathrm{avrA}^{-}\right)$detected in mucosal or lymphoid tissues following infection with this strain. The elevated numbers of translocated SL3201(avrA 7 ) plausibly migrate unassociated with immune cells along efferent drainage vessels to the MLN and then disseminate to the spleen. These observations are reminiscent of systemic bacterial infections mediated by Yesinia pestis, where YopJ/P selectively kills the infected macrophages, allowing rapid extracellular proliferation and dissemination. Thus, AvrA activity may account for the self-limited inflammation seen in Salmonellosis. The AvrA protein allows the invading bacterium to dampen innate immune signaling, while also preventing the apoptotic elimination of its host cell niche. These data suggest AvrA, by suppressing apoptosis, does indeed act as a true avirulence factor, in that its absence results in enhanced disease at the whole organism level.

\section{EXPERIMENTAL PROCEDURES}

\section{Bacterial Strains}

Isogenically matched strains of Salmonella enterica serovar typhimurium SL3201 wild type, $a v r A^{-}$mutant strain, and $a v r A^{-}$mutant strain complemented with a plasmid expressing AvrA (avrA ${ }^{-}$pAvrA) were described earlier (Collier-Hyams et al., 2002). Salmonella 
strains were inoculated in LB broth containing $25 \mu \mathrm{g} \mathrm{ml}^{-1}$ nalidixic acid and/or $100 \mu \mathrm{g} \mathrm{ml}^{-1}$ ampicillin, and cultured in a shaker for 6 to $8 \mathrm{~h}$ at $37^{\circ} \mathrm{C}$. The cultures were then diluted $1 / 1000$ in $40 \mathrm{ml}$ of $\mathrm{LB}$ broth with antibiotics and grown $16 \mathrm{~h}$ at $37^{\circ} \mathrm{C}$ under non-agitated microaerophilic conditions as previously described (Vijay-Kumar et al., 2006).

\section{Mouse experiments}

6 to 8 weeks old pathogen-free female BALB/cj mice were purchased from Jackson Laboratories (Bar Harbor, ME). Food and water were withdrawn $4 \mathrm{~h}$ before treatment with $7.5 \mathrm{mg}$ of streptomycin by oral gavage. Animals were deprived of food and water for 4 hagain at $20 \mathrm{~h}$ post streptomycin treatment; then the mice were infected with $10^{8} \mathrm{CFU}$ wild type or mutant Salmonella SL3201. The quantity of bacterial inoculum was further confirmed by plate count. At designated intervals post infection (p.i.), blood samples were collected and serum KC levels were determined using a mouse CXC chemokine KC ELISA kit according to the manufacturer's instructions (R\&D Systems, Minneapolis, MN). Thereafter, mice were sacrificed, ceca were removed and neutrophil infiltration into tissue quantified by measuring myeloperoxidase (MPO) enzyme activity (a marker for neutrophils) by biochemical assay. Histological sections of ceca were prepared from 5 infected animals per treatment. Cecal sections were assessed using a clinical index score previously described (Vijay-Kumar et al., 2006). Animal experiments were approved by the Emory University institutional ethical committee and performed according to the legal requirements.

\section{Cytotoxicity Assay}

Lactate dehydrogenase (LDH) activity in cell culture medium (a gauge of cytotoxicity) was measured with a CytoTox $96^{\circledR}$ Non-Radioactive Cytotoxicity Assay kit (Promega Corporation, Madison, WI) according to manufacturer's instructions. Cells were seeded in 24- or 48-well tissue culture plate, and treated with bacteria or chemical reagents for the indicated time period. Percentage cytotoxicity is expressed as sample $\mathrm{LDH} /$ maximum LDH $\times 100 \%$.

\section{Adenovirus propagation}

Replication defective adenoviral delivery vectors expressing Salmonella enterica serovar typhimurium AvrA (Ad-AvrA), mutant AvrA (Ad-mAvrA) or green fluorescent protein under the transcriptional control of the cytomegalovirus immediate-early promoter were constructed using the AdEasy XL Adenoviral Vector System (Stratagene, La Jolla, CA). The mutant AvrA, mAvrA, containing cysteine to alanine replacement at position 186 (C186A) is catalytically inactive (Collier-Hyams et al., 2002). Recombinant adenoviral plasmids were transfected into AD-293 cells to form primary recombinant viruses. The recombinant adenoviruses were amplified in AD-293 cells and purified by AdEasy Virus Purification Kit (Stratagene). Biosafety of experiments using recombinant adenovirus was performed according to the manufacturer's guidelines.

\section{Bone marrow derived macrophages}

Mouse femurs and tibias were retrieved, and adherent tissues were separated. The ends of the bones were cut off, and marrow was flushed out with Hank's media. Cells were filtered through cell strainer $(40 \mu \mathrm{m})$, and washed again with Hank's media. Bone marrow cells were cultured in DMEM with $10 \%$ FCS and $10 \mathrm{ng} / \mathrm{ml}$ of M-CSF at $37^{\circ} \mathrm{C}$ in a humidified $5 \%$ $\mathrm{CO}_{2}$ atmosphere. At day 5, culture media was replaced by fresh media supplemented with M-CSF. 


\section{Antibodies and Reagents}

Phospho-JNK, cleaved caspase 3, and cleaved PARP antibodies were purchased from Cell Signaling Technology (Danvers, MA) and anti-c-myc from Clontech (Mountain View, CA). Anti- $\beta$-actin antibody and actinomycin D were obtained from Sigma (St. Louis, MO). Recombinant human or rat TNF $\alpha$ was purchased from R\&D Systems (Minneapolis, MN), and Recombinant murine TNF- $\alpha$ from Peprotech Inc. (Rocky Hill, New Jersey).

\section{TUNEL assay}

Apoptosis was examined by the TUNEL assay in paraffin embedded slides using ApopTag Plus Peroxidase In Situ Apoptosis Detection Kit (Milipore, St. Charles, MO) according to the manufacturer's instructions. Apoptotic cells were counted from 10 continuous fields $(400 \mathrm{x}) /$ slide.

\section{Flow Cytometry}

Single cell suspensions were prepared from the spleens of mice infected with Salmonella strains. The spleens were homogenized and RBCs lysed. Splenocytes were first incubated with specific fluorescent antibodies for cell-surface markers. Fixed and permeabilized cells were stained with an antibody against Salmonella (KPL, Inc., Gaithersburg, MD). Samples were acquired on a FACSCalibur (BD Biosciences), and data were analysed using FlowJo software (Tree Star, Inc.); 250000 events were collected. Anti-F4/80, CD11b antibodies were purchased from BD Biosciences.

\section{Immunoblot assays}

Cell lysates were routinely collected in ice-cold buffer containing $50 \mathrm{mM}$ Tris. $\mathrm{HCl}(\mathrm{pH} 7.5)$, $150 \mathrm{mM} \mathrm{NaCl}, 1 \%$ Nonidet P-40, $0.5 \%$ sodium deoxycholate, $0.1 \%$ SDS, and protease inhibitor cocktail (Roche, Florence, SC). The protein concentration of each sample was determined using the Bradford reagent (Bio-Rad; Hercules, CA). Equal amounts of protein were loaded on SDS-PAGE and blotted with indicated antibodies.

\section{Statistical Analysis}

Differences between multiple groups were analyzed by one-way or two-way analysis of variance (ANOVA) followed by Bonferroni's Multiple Comparison Test using GraphPad Prism version 5; show as $* \mathrm{p}<0.05$, ** $\mathrm{p}<0.01$, and $* * * \mathrm{p}<0.001$. Values are expressed as the mean \pm the standard error.

\section{Supplementary Material}

Refer to Web version on PubMed Central for supplementary material.

\section{Acknowledgments}

This work was supported by the Burroughs Wellcome Foundation and NIH DK71604 AI644662 (ASN), and R.M.J. is supported by NIH grants R03DK089304 and K01DK081481. The authors have no financial conflict of interest.

\section{References}

Barthel M, Hapfelmeier S, Quintanilla-Martinez L, Kremer M, Rohde M, Hogardt M, et al. Pretreatment of mice with streptomycin provides a Salmonella enterica serovar Typhimurium colitis model that allows analysis of both pathogen and host. Infect Immun. 2003; 71:2839-2858.

[PubMed: 12704158] 
Collier-Hyams LS, Zeng H, Sun J, Tomlinson AD, Bao ZQ, Chen H, et al. Cutting edge: Salmonella AvrA effector inhibits the key proinflammatory, anti-apoptotic NF-kappa B pathway. J Immunol. 2002; 169:2846-2850. [PubMed: 12218096]

Du F, Galan JE. Selective inhibition of type III secretion activated signaling by the Salmonella effector AvrA. PLoS Pathog. 2009; 5:e1000595. [PubMed: 19779561]

Duesbery NS, Webb CP, Leppla SH, Gordon VM, Klimpel KR, Copeland TD, et al. Proteolytic inactivation of MAP-kinase-kinase by anthrax lethal factor. Science. 1998; 280:734-737. [PubMed: 9563949]

Ellis JG, Dodds PN, Lawrence GJ. The role of secreted proteins in diseases of plants caused by rust, powdery mildew and smut fungi. Curr Opin Microbiol. 2007; 10:326-331. [PubMed: 17698407]

Fehr D, Casanova C, Liverman A, Blazkova H, Orth K, Dobbelaere D, et al. AopP, a type III effector protein of Aeromonas salmonicida, inhibits the NF-kappaB signalling pathway. Microbiology. 2006; 152:2809-2818. [PubMed: 16946275]

Glomski IJ, Decatur AL, Portnoy DA. Listeria monocytogenes mutants that fail to compartmentalize listerolysin $\mathrm{O}$ activity are cytotoxic, avirulent, and unable to evade host extracellular defenses. Infect Immun. 2003; 71:6754-6765. [PubMed: 14638761]

Hardt WD, Galan JE. A secreted Salmonella protein with homology to an avirulence determinant of plant pathogenic bacteria. Proc Natl Acad Sci U S A. 1997; 94:9887-9892. [PubMed: 9275221]

Jones RM, Neish AS. Recognition of bacterial pathogens and mucosal immunity. Cell Microbiol. 2011; 13:670-676. [PubMed: 21352463]

Jones RM, Wu H, Wentworth C, Luo L, Collier-Hyams L, Neish AS. Salmonella AvrA Coordinates Suppression of Host Immune and Apoptotic Defenses via JNK Pathway Blockade. Cell Host Microbe. 2008; 3:233-244. [PubMed: 18407067]

Jones RM, Sloane VM, Wu H, Luo L, Kumar A, Kumar MV, et al. Flagellin administration protects gut mucosal tissue from irradiation-induced apoptosis via MKP-7 activity. Gut. 2011; 60:648-657. [PubMed: 21199832]

Li H, Xu H, Zhou Y, Zhang J, Long C, Li S, et al. The phosphothreonine lyase activity of a bacterial type III effector family. Science. 2007; 315:1000-1003. [PubMed: 17303758]

Mittal R, Peak-Chew SY, McMahon HT. Acetylation of MEK2 and I kappa B kinase (IKK) activation loop residues by YopJ inhibits signaling. Proc Natl Acad Sci U S A. 2006; 103:18574-18579. [PubMed: 17116858]

Monack DM, Mueller A, Falkow S. Persistent bacterial infections: the interface of the pathogen and the host immune system. Nat Rev Microbiol. 2004; 2:747-765. [PubMed: 15372085]

Monack DM, Hersh D, Ghori N, Bouley D, Zychlinsky A, Falkow S. Salmonella exploits caspase-1 to colonize Peyer's patches in a murine typhoid model. J Exp Med. 2000; 192:249-258. [PubMed: 10899911]

Mukherjee S, Hao YH, Orth K. A newly discovered post-translational modification--the acetylation of serine and threonine residues. Trends Biochem Sci. 2007; 32:210-216. [PubMed: 17412595]

Mukherjee S, Keitany G, Li Y, Wang Y, Ball HL, Goldsmith EJ, Orth K. Yersinia YopJ acetylates and inhibits kinase activation by blocking phosphorylation. Science. 2006; 312:1211-1214. [PubMed: 16728640]

Orth K, Palmer LE, Bao ZQ, Stewart S, Rudolph AE, Bliska JB, Dixon JE. Inhibition of the mitogenactivated protein kinase kinase superfamily by a Yersinia effector. Science. 1999; 285:1920-1923. [PubMed: 10489373]

Ruckdeschel K, Roggenkamp A, Lafont V, Mangeat P, Heesemann J, Rouot B. Interaction of Yersinia enterocolitica with macrophages leads to macrophage cell death through apoptosis. Infect Immun. 1997; 65:4813-4821. [PubMed: 9353070]

Sansonetti PJ, Di Santo JP. Debugging how bacteria manipulate the immune response. Immunity. 2007; 26:149-161. [PubMed: 17307704]

Staskawicz BJ, Mudgett MB, Dang1 JL, Galan JE. Common and contrasting themes of plant and animal diseases. Science. 2001; 292:2285-2289. [PubMed: 11423652]

Tam MA, Rydstrom A, Sundquist M, Wick MJ. Early cellular responses to Salmonella infection: dendritic cells, monocytes, and more. Immunol Rev. 2008; 225:140-162. [PubMed: 18837781] 
Trosky JE, Li Y, Mukherjee S, Keitany G, Ball H, Orth K. VopA inhibits ATP binding by acetylating the catalytic loop of MKKs. J Biol Chem. 2007

Trosky JE, Mukherjee S, Burdette DL, Roberts M, McCarter L, Siegel RM, Orth K. Inhibition of MAPK signaling pathways by VopA from Vibrio parahaemolyticus. J Biol Chem. 2004; 279:51953-51957. [PubMed: 15459200]

Vijay-Kumar M, Wu H, Jones R, Grant G, Babbin B, King TP, et al. Flagellin suppresses epithelial apoptosis and limits disease during enteric infection. Am J Pathol. 2006; 169:1686-1700. [PubMed: 17071592]

Weinrauch Y, Zychlinsky A. The induction of apoptosis by bacterial pathogens. Annu Rev Microbiol. 1999; 53:155-187. [PubMed: 10547689] 
A
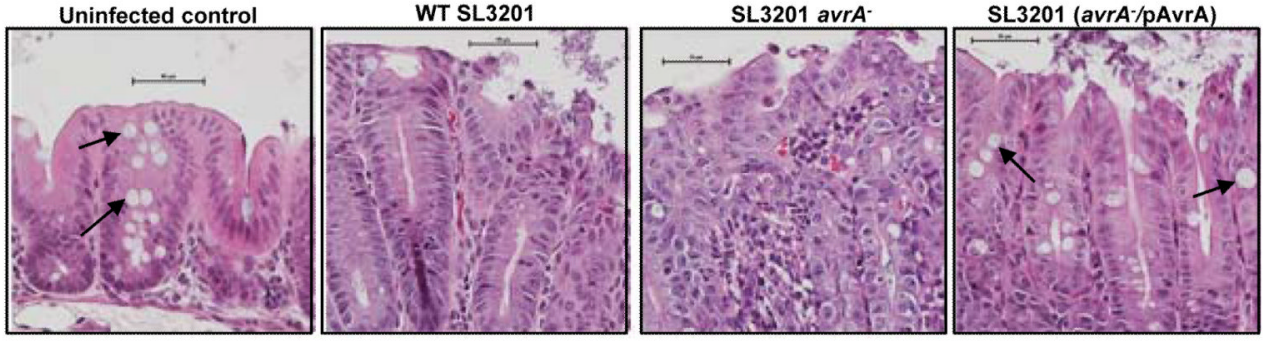

$\mathbf{B}$
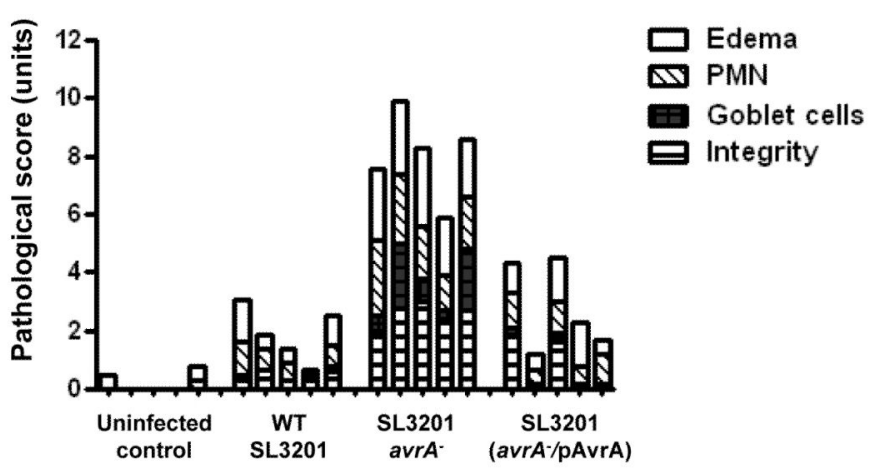

C

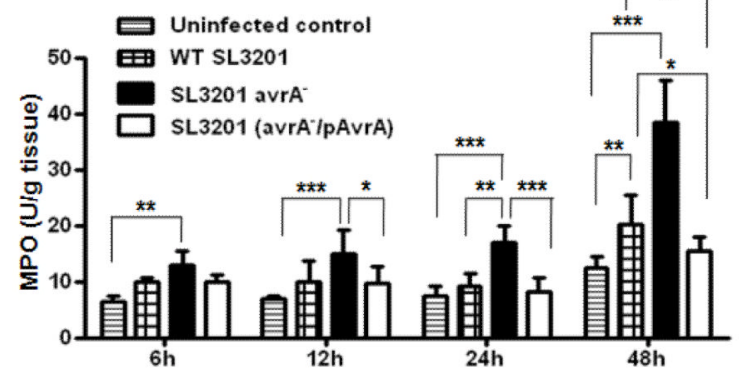

Fig. 1. Natural infection with mutant Salmonella SL3201 (avrA $\left.{ }^{-}\right)$induces a more severe inflammatory response

A. H \& E staining of cecal mucosa sections from mice at 24 hours following oral infection with the indicated Salmonella genotypes. Scale bar: $50 \mu \mathrm{m}$. Arrow: goblet cells.

B. Semi-quantitative inflammatory scores of murine cecal mucosa from H \& E staining of sections 24 h p.i. displayed in A. Edema, infiltration of polymorphonuclear cell, decrease of goblet cell numbers, and integrity of epithelial layer were scored separately.

C. Cecum MPO (myeloperoxidase) activity in mice orally infected with the indicated Salmonella geneotypes at 6, 12, 24, or 48 hours p.i.. Error bars represent Standard Error $(\mathrm{n}=5)$. Data were analyzed first using Two way ANOVA, then Bonferroni post test $(*$ $\mathrm{P}<0.05$, ** $\mathrm{P}<0.01$, *** $\mathrm{P}<0.001)$. 
A
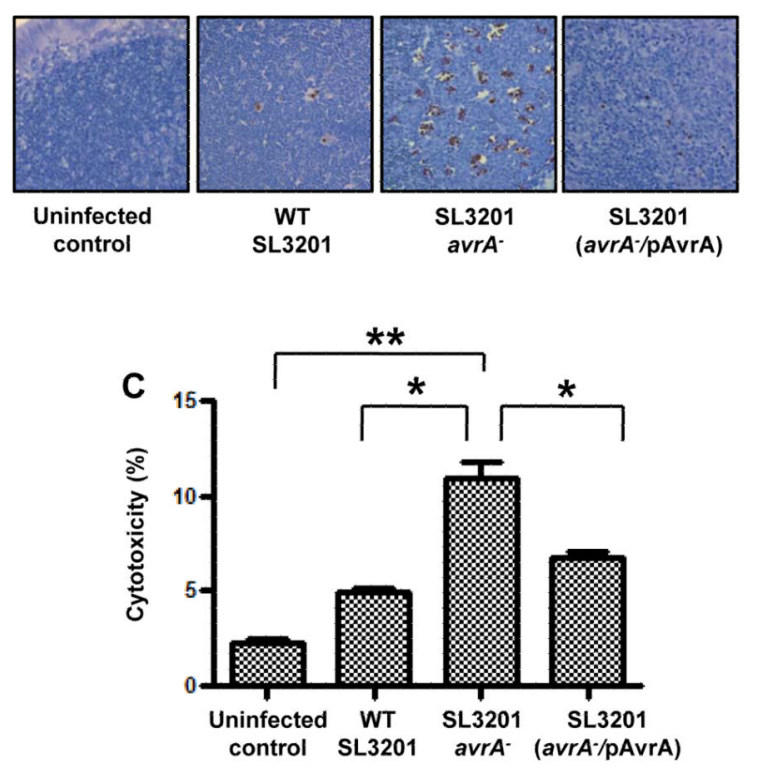
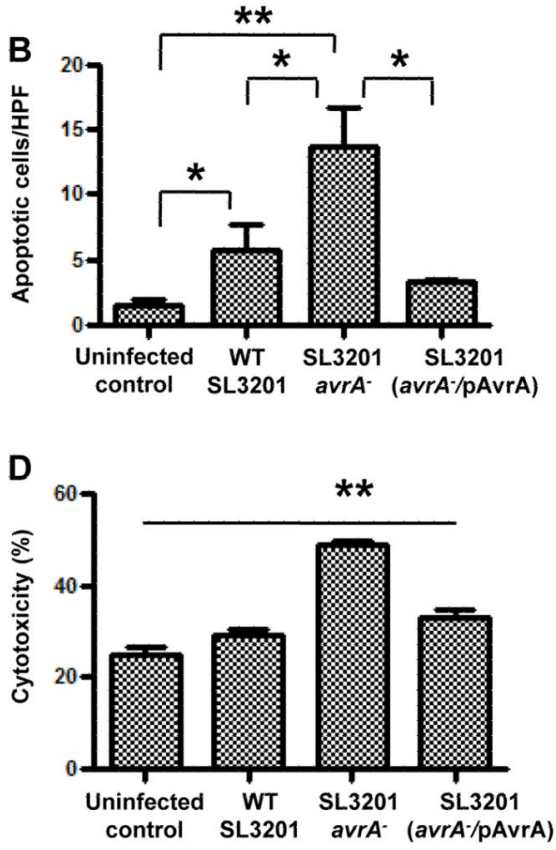

Fig. 2. Salmonella secreted protein AvrA dampens apoptosis in multiple murine tissues following infection

A. Histochemical apoptotic (TUNEL assay) staining of cecal patch sections from mice infected with the indicated Salmonella genotypes for 24 hours.

B. Enumeration of apoptotic cells per high power field (HPF) in figure $2 A(n=5)$. C. Quantification of lactate dehydrogenase ( $\mathrm{LDH})$ activity in culture supernates of ex vivo mouse bone marrow derived macrophage cells following infection with indicated Salmonella genotypes at multiplicity of infection (m.o.i.) of $10(n=3)$.

D. Quantification of LDH activity in culture supernates of primary epithelial cells (IEC-6) following infection with indicated Salmonella genotypes at a m.o.i. of $10(n=3)$. One way ANOVA and Bonferroni post test $(* \mathrm{p}<0.05, * * \mathrm{P}<0.01)$. 
A

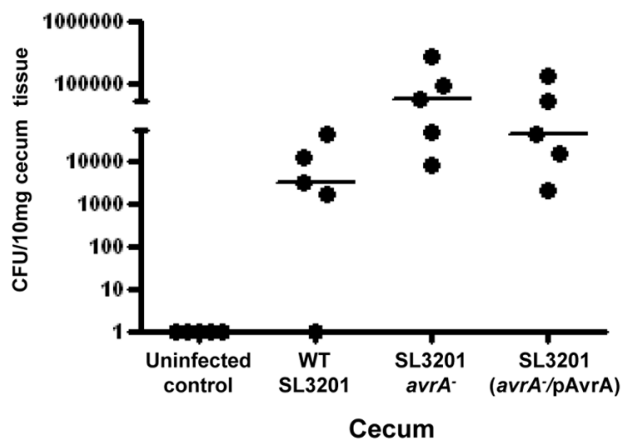

C

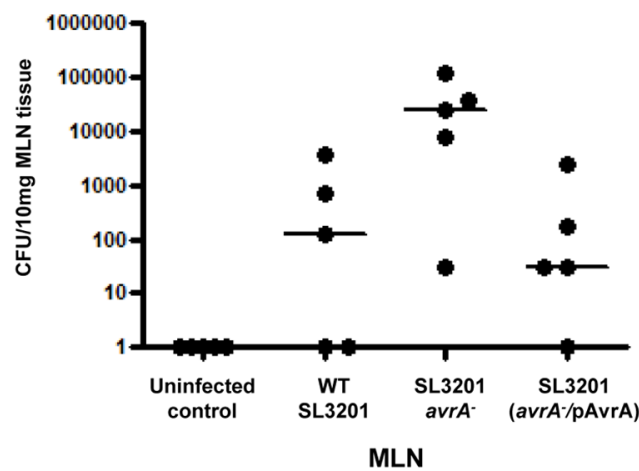

E

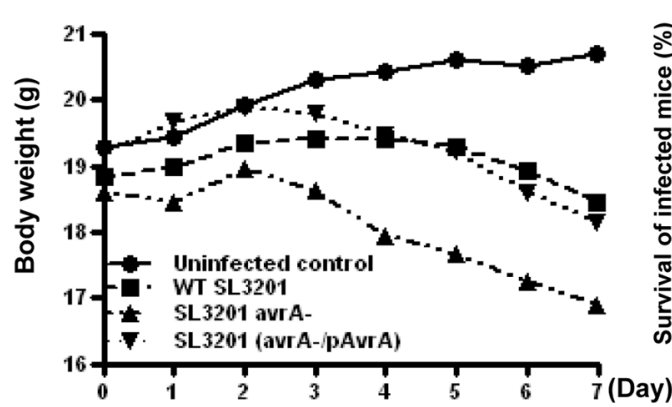

G

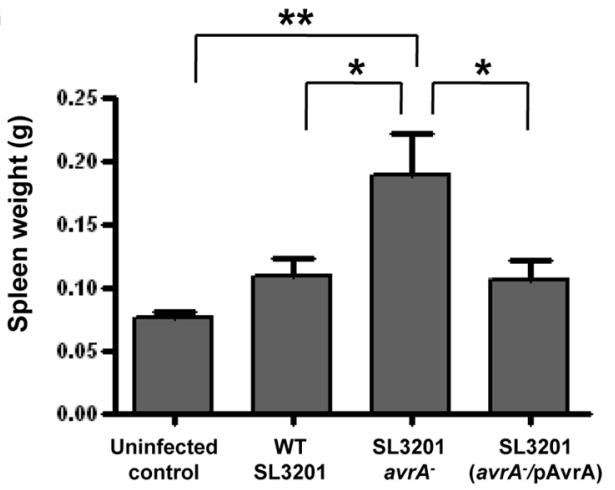

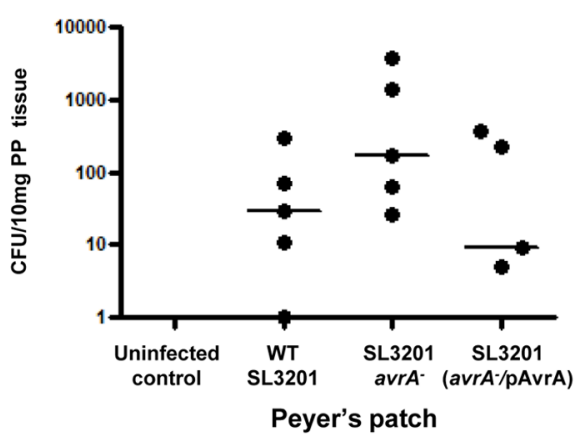

D

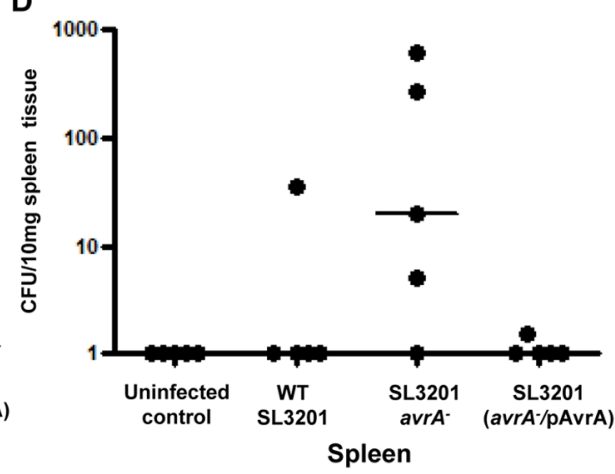

$\mathbf{F}$
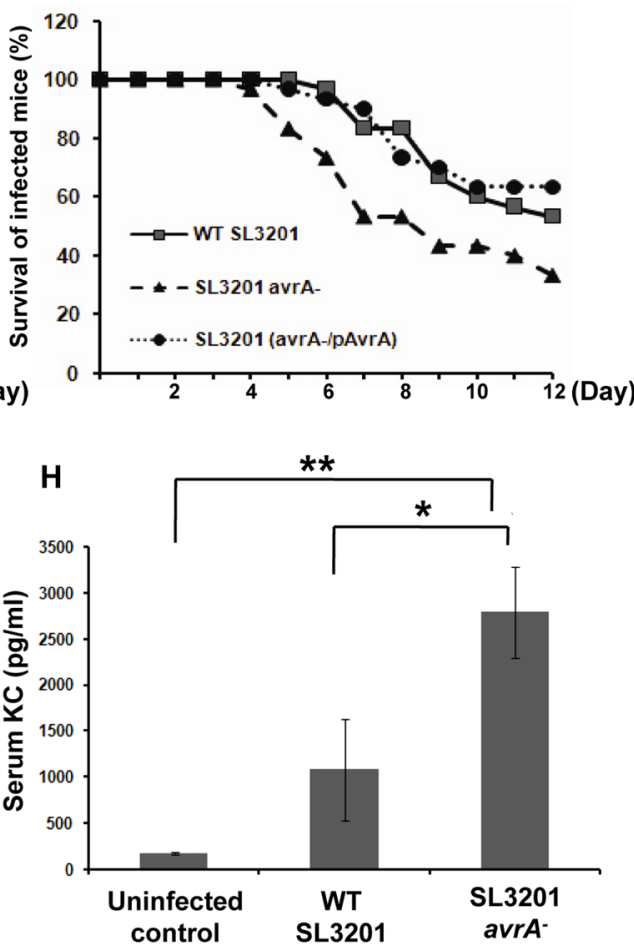

Fig. 3. Salmonella SL3201 ( $\operatorname{avr}^{-}$) exhibited increased mucosal and systemic dissemination in vivo 
A. Enumeration of Salmonella in the cecum of mice at 12 hours following oral infection with indicated Salmonella genotypes. Wild type and $a v r A^{-}$Salmonella were counted following selective propagation on LB agar media plates containing $25 \mu \mathrm{g} / \mathrm{ml}$ nalidixic acid, and $a v r A^{-} / \mathrm{pAvrA}$ was propagated on LB agar media plates containing $25 \mu \mathrm{g} / \mathrm{ml}$ nalidixic acid, and ampicillin $100 \mu \mathrm{g} / \mathrm{ml},(\mathrm{n}=5)$.

B. Enumeration of Salmonella in the peyer's patch of mice at $6 \mathrm{~h}$ following oral infection with indicated Salmonella genotypes, using selective media as described in $3 \mathrm{~A},(\mathrm{n}=5)$.

C. Enumeration of Salmonella in the mesenteric lymph node (MLN) of mice $12 \mathrm{~h}$ following oral infection with indicated Salmonella genotypes, using selective media as described in $3 \mathrm{~A},(\mathrm{n}=5)$.

D. Enumeration of Salmonella in the spleen of mice at $24 \mathrm{~h}$ following oral infection with indicated Salmonella genotypes, using selective media as described in $3 \mathrm{~A},(\mathrm{n}=5)$.

E. Body weight loss in mice infected with the indicated Salmonella genotypes, $(\mathrm{n}=15)$.

F. Mortality in mice infected with the indicated Salmonella genotypes, $(\mathrm{n}=5)$.

G. Spleen weight in mice infected with the indicated Salmonella genotypes for 7 days, $(\mathrm{n}=5)$.

H. Serum KC in mice infected with the indicated Salmonella genotypes, ( $\mathrm{n}=5)$. 
A

A

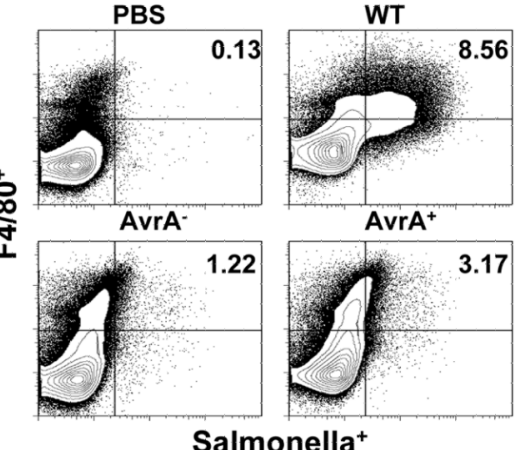

B

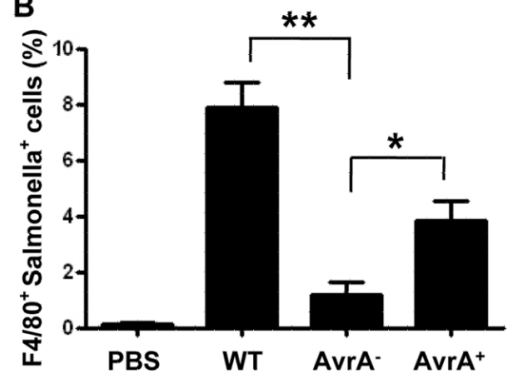

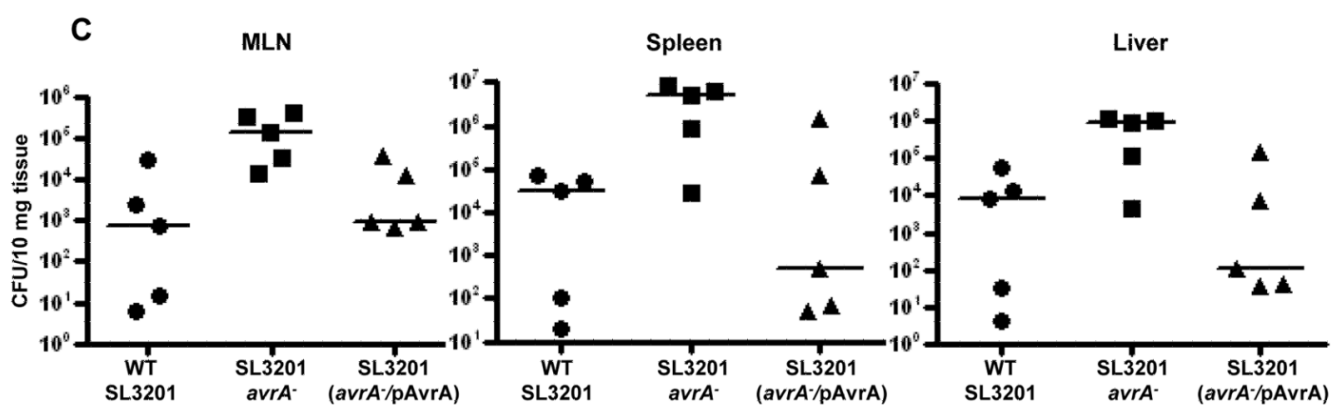

D

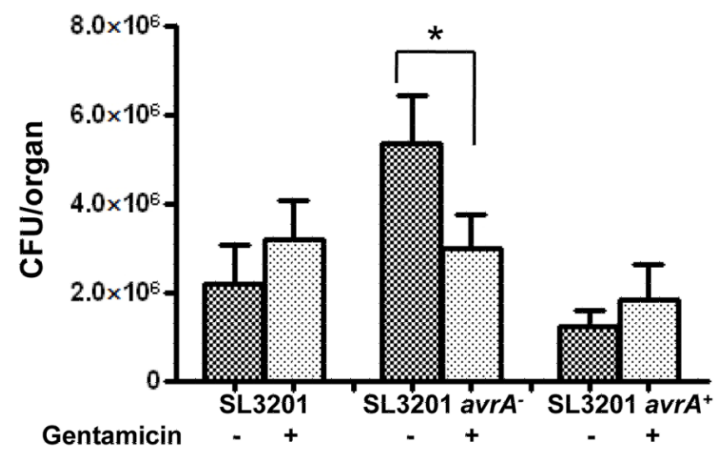

Fig. 4. SL3201 $\left(\mathrm{avrA}^{-}\right)$does not establish an intracellular niche within macrophages A. Flow cytometric analysis of splenocyte populations in infected mice.

B. Enumeration of Salmonella and F4/80 double positive cell populations 6 days following oral infection with indicated Salmonella genotypes detected in A, $(\mathrm{n}=4)$. This experiment was repeated three times with essentially identical results. $(* \mathrm{P}<0.05, * * \mathrm{P}<0.01)$

C. Enumeration of Salmonella in the MLN, spleen and liver of mice on the $6^{\text {th }}$ day following oral infection with indicated Salmonella genotypes $(\mathrm{n}=5)$.

D. Three groups of mice ( $\mathrm{n}=10$ /group) were feeded orally with $1.03 \times 10^{8} \mathrm{CFU}$ of bacteria for five days. $6 \mathrm{~h}$ before mice were sacrificed, half of the mice were injected subcutaneously with $1 \mathrm{mg} /$ mouse of gentamicin ( $\mathrm{n}=5 /$ group); then spleens were removed and spread on the plates with Nalidixic Acid and/or Ampicillin to analysis the bacterial colonization. 

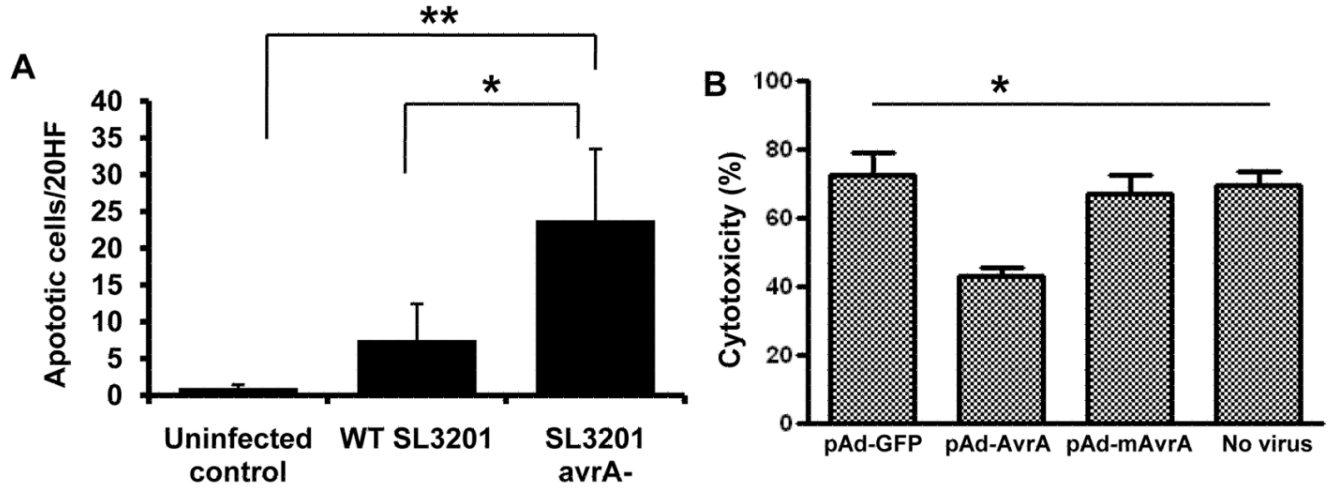

C

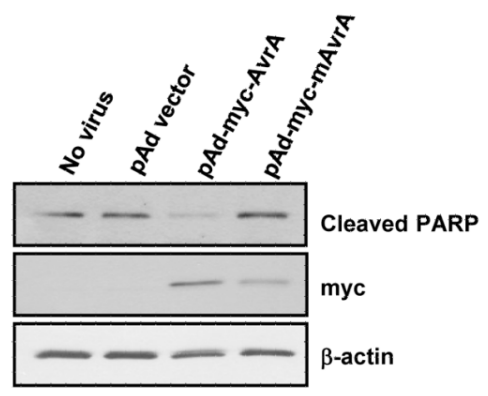

D

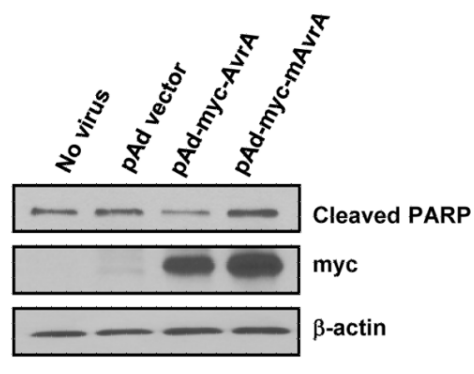

Fig. 5. AvrA directly suppresses induced apoptosis

A. Enumeration of cleaved caspase 3 positive apoptotic cells in spleen at day 7 p.i..

B. Lactate dehydrogenase (LDH) activity in culture supernates of primary epithelial cells (IEC-6) transduced with the indicated virus particles before stimulation with actinomycin D and $\mathrm{TNF} \alpha$ for 24 hours.

C. Immunoblot analysis of lysates from primary epithelial cells (IEC-6) transduced with the indicated virus particles before stimulation with actinomycin D and TNF $\alpha$ for 24 hours, using an antibody against cleaved PARP or myc-tag.

D. Immunoblot analysis of lysates from cultured murine bone marrow derived macrophages transduced with the indicated virus particles described in Fig 5B, before stimulation with actinomycin-D and TNF $\alpha$ for 24 hours, using an antibody against cleaved PARP or myc-tag. 